MATEC Web of Conferences 25,02010

(2015)

DOI: $10.1051 /$ matec conf/ 20152502010

(C) Owned by the authors, published by EDP Sciences, 2015

\title{
Shrinkage Simulation of Holographic Grating Using Diffusion Model in PQ-PMMA Photopolymer
}

\author{
Zepeng Wei, Dan Yu*, Xiaolan Li, Yanwen Wang, Zhuang Chen, Yaohui Geng \& Jiang Lv \\ China College of Sciences, Tianjin University of Technology, Tianjin, China
}

\begin{abstract}
An extended model based on nonlocal polymerization-driven diffusion model is derived by introducing shrinkage process for describing photopolymerized dynamics in PQ-PMMA photopolymer. The kinetic parameters, polymerization rate and diffusion rate are experimentally determined to provide quantitative simulation. The numerical results show that the fringes at edge of grating are firstly shifted and consequently, it leads to a contrast reduction of holograms. Finally, theoretical results are experimentally checked by temporal evolution of diffraction efficiency, and the shrinkage coefficient $0.5 \%$ is approximately achieved under incident intensity $25.3 \mathrm{mw} / \mathrm{cm} 2$. This work can enhance the applicability of diffusion model and contribute to the reasonable description of the grating formation in the photopolymer.
\end{abstract}

Keywords: photopolymer; holographic grating; diffusion model; shrinkage

\section{INTRODUCTION}

The holographic data storage is considered as one of next generation information storage technologies due to their high density and large capacity ${ }^{1,2}$. In recent years, the interest in photopolymer material as promising candidate for holographic memory has increased dramatically ${ }^{3,4}$. Photopolymer materials have much attention for use in holography, mainly because they can be designed to have high diffraction efficiency, real-time processing and they are low cost and easily synthesized and so on ${ }^{5,6}$. Unfortunately, most photopolymers exhibit photoinduced shrinkage effect, which lead to the limitation in commercial applications ${ }^{7,8}$. Here, the crucial reasons can be ascribed to the dimensional and bulk refractive index modulation changes induced by radical photopolymerization, so that the recorded refractive index grating has different grating space from that of the light interference fringe.

Shrinkage in photopolymers has always been a significant consideration in holographic data storage and optical elements. So far, several models have been presented to describe the volume shrinkage, in which fringe-plane rotation geometry and reciprocal space (K-sphere) are general methods for analyzing this effect $^{9-13}$. However, there are just a few simply geometry approaches, which leaded to neglecting the realistic photochemical processes. In order to reflect the realistic physical mechanism involved, we will describe the shrinkage effect using a non-local polymerization drive diffusion model (NPDD). The NPDD model is an effective approach in analyzing the photochemical dynamics of photopolymers, because the monomer and photoproducts are introduced into non-local area ${ }^{14}$. In previous literatures, the NPDD model was used to describe the dynamics of grating formation based on constant fringe space ${ }^{15}$. However,

*Corresponding author: yudanhit@,126.com the fringe space is actually changed during recording exposure due to volume shrinkage. In this paper, we proposed an extended model based on nonlocal diffusion model to describe the photopolymerized shrinkage dynamics in PQ-PMMA polymer. The main feature of the model is that the temporal evolution of shrinkage coefficient which leads to the change of grating space is introduced. The model is then solved numerically to describe the shrinkage dynamics and the reasonable results are obtained.

\section{MATERIALS AND EXPERIMENTAL SETUP}

In our experiments, the samples were formed by poly (methyl methacrylate) (PMMA) host matrix and phenanthrenequinone (PQ) photosensitizer. The thermal initiator 2, 2-azobis (2-methlpropionitrile) (AIBN) and PQ molecules were dissolved in a solvent methyl methacrylate (MMA) and mixed to form a uniform solution. The mixture was poured into a glass mold and solidified at $60^{\circ} \mathrm{C}$ for $120 \mathrm{~h}$. After the thermal polymerization, the sample with PQ's concentration $0.1 \mathrm{M}$ and $2 \mathrm{~mm}$ thickness was prepared.

Two-beam coupling experimental setup was used to investigate the dark enhancement of holographic gratings, as shown in Figure 1. A continuous-wave diode pumped solid-state laser with $532 \mathrm{~nm}$ wavelength is used as a recording light source. Two s-polarized recording beams with equal intensities and an intersection outside angle of $60^{\circ}$ entered the sample. A He-Ne laser at $633 \mathrm{~nm}$ incident on the sample at Bragg's angle was used for nondestructive real time detection of diffraction efficiency. The sample was placed on a computer-controlled motorized stage with a resolution of $0.0025^{\circ}$. By rotating the sample, the angle selectivity curve was measured. 


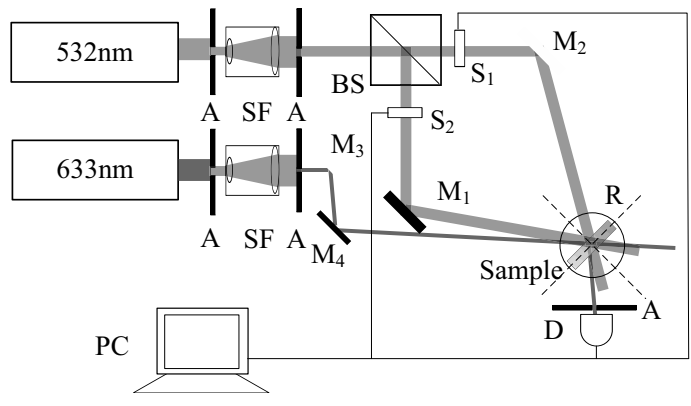

Figure 1. Experimental setup for recording transmittance gratings in the sample (A1, A2: apertures; BS: beam-splitter; M1-M4: mirrors; R: rotation; D: detector)

\section{DETERMINATION OF KINETICS PARAME- TERS}

The determination of kinetic parameters, polymerization rate and diffusion rate, is crucial for investigating the photochemical dynamics in the photopolymer ${ }^{16}$. However, no value was available for this polymer in the literatures to our knowledge, especially the dependence of polymerization rate on the incident intensity. To obtain the order of photochemical reaction, the temporal evolution of diffraction efficiency were measured with various incident irradiances. We can compute the estimate of the slope of $\arcsin (\sqrt{ } \eta)$ using finite differences, similar as description in reference ${ }^{17}$ :

$\frac{\mathrm{d} \arcsin \sqrt{\eta}}{\mathrm{d} t}=\frac{\pi d}{\lambda \cos \theta} \frac{\mathrm{d} \Delta n(t)}{\mathrm{d} t}=\frac{C_{0} \pi d}{\lambda \cos \theta} \kappa I_{0}^{\gamma} \propto I_{0}^{\gamma}(1)$

The order of photochemical reaction $\gamma=1.03$ can be determined by nonlinear fitting of this curve, as shown in Figure 1(a). It implies that the dependence of polymerization rate on the incident irradiance is nearly linear.

The polymerization is a primary photochemical process at the beginning of the grating formation. It is assumed that the refractive index modulation is linearly related to the polymer concentration at this process. The refractive index modulation can be approximately expressed ${ }^{18}$.

$$
\Delta n(t)=C_{0}[\mathrm{PQ}]_{0}\left[1-\exp \left(-\kappa I_{0} t\right)\right]
$$

The polymerization rate parameter can be estimated with nonlinear fitting the initial step of grating formation, which is shown in inset of Figure 2(a). The typical characteristic parameter $\kappa=3.46 \times 10^{-5}$ $\mathrm{cm}^{2} \mathrm{~mW}^{-1} \mathrm{~s}^{-1}$ is extracted at $25.3 \mathrm{mw} / \mathrm{cm}^{2}$. Figure 2(b) shows the dark enhancement of diffraction efficiency after short exposure time (100s). The curve is fitted using the exponential function, and the diffusion coefficient $D=4.2 \times 10^{-18} \mathrm{~m}^{2} / \mathrm{s}$ of PQ's concentration is obtained based on the relation $\tau=1 /\left(D K^{2}\right)$.
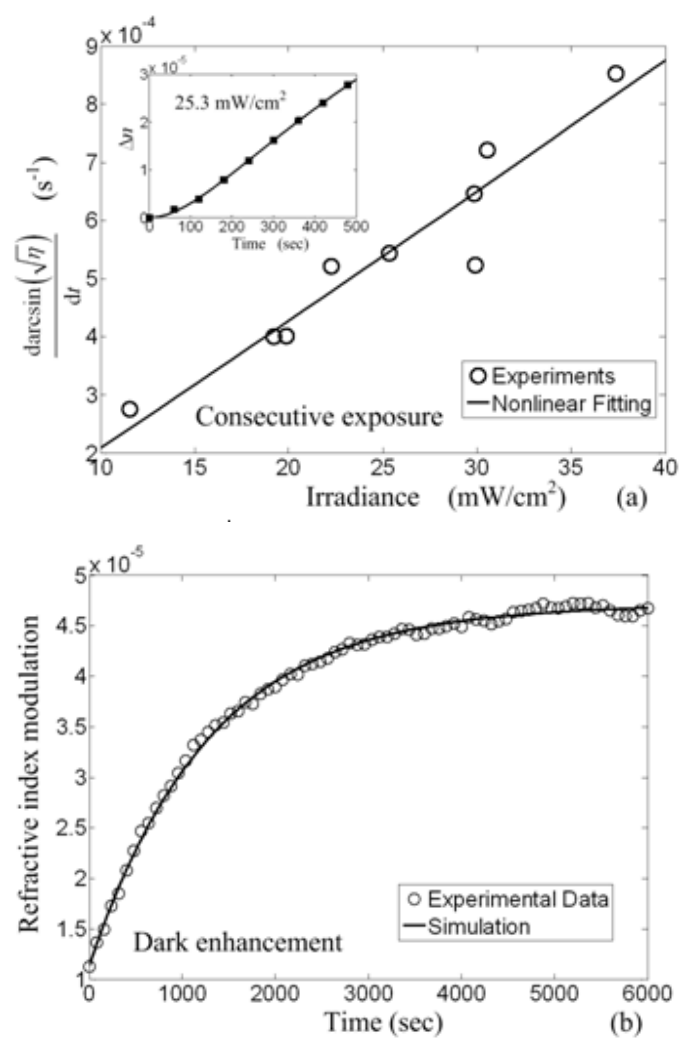

Figure 2. (a) darcsin $(\sqrt{ } \eta) / d t$ as a function of irradiance. The inset is initial step of grating formation by nonlinear fitting. (b) Dark enhancement of grating after recording exposure fitted by exponential function.

\section{SHRINKAGE SIMULATION}

\subsection{An extended diffusion model}

The polymerization process is always accompanied with a thickness variation of the sample ${ }^{11}$. The growth evolution of polymer density corresponds to the thickness shrinkage of the sample. There is reasonable estimation that the thickness variation is proportional to the degree of polymerization reaction. Consequently, a temporal evolution of thickness variation and polymer density can be described by equivalent asymptotic curve, similar to reference ${ }^{16}$ :

$\Delta d(t)=\Delta d_{\max }\left[1-\exp \left(-\kappa I_{0} t\right)\right]$

The corresponding shrinkage coefficient can be given by

$\rho(t)=\frac{\Delta d(t)}{d_{0}} \times 100 \%=\rho_{0}\left[1-\exp \left(-\kappa I_{0} t\right)\right]$

Where, $d_{0}$ is thickness of sample. The dimensional 
change is assumed to take place in the out-of-plane direction due to the bulk photopolymer. A fractional change in thickness will result in changing the tilt angle and grating spacing, which can be derived as ${ }^{9}$ :

$\phi_{1}=\frac{\pi}{2}-\arctan \left[\frac{\tan \left(\pi / 2-\phi_{0}\right)}{1-\rho}\right]$

$\Lambda_{1}=\Lambda_{0} \frac{\sin \phi_{1}}{\sin \phi_{0}}$

Where, $\phi_{0}, \Lambda_{0}$, and $\phi_{1}, \Lambda_{1}$ are respectively the tilt angle and the fringe grating before and after dimensional change of sample.

In our paper nonlocal polymerization driven diffusion (NPDD) model which is used to simulate the shrinkage process can be written as ${ }^{14}$ :

$\frac{\partial u(x, t)}{\partial t}=\frac{\partial}{\partial x}\left[D \frac{\partial u(x, t)}{\partial x}\right]-\int_{-\infty}^{+\infty} R\left(x, x^{\prime}\right) F\left(x^{\prime}\right) u\left(x^{\prime}, t\right) \mathrm{d} x^{\prime}$

Where $u(x, t)$ is the PQ's concentration and $D$ is the diffusion coefficient. The polymerization rate can be depicted as follows:

$$
F(x)=\kappa I_{a}^{\gamma}[1+\mathrm{V} \cos (K x)]^{\gamma}
$$

Where $\mathrm{V}$ is the visibility and the absorbed intensity $I_{a}=I_{0}[1-\exp (-\varepsilon d[\mathrm{PQ}])]^{18}$. Here, the linear relation between the polymerization rate and the irradiance is used. The nonlocal response function $R\left(x, x^{\prime}\right)$ can be expressed as:

$R\left(x, x^{\prime}\right)=\frac{1}{\sqrt{2 \pi \sigma}} \exp \left[\frac{-\left(x-x^{\prime}\right)^{2}}{2 \sigma}\right]$

Where, $\sigma^{1 / 2}$ represents the nonlocal response length. After exposed for time $t$, the concentration of polymerized PQ molecules at location $x$ can be given as:

$$
P(x, t)=\int_{0}^{t} \int_{-\infty}^{+\infty} R\left(x, x^{\prime}\right) F\left(x^{\prime}\right) u\left(x^{\prime}, t^{\prime}\right) \mathrm{d} x^{\prime} \mathrm{d} t^{\prime}
$$

Due to low shrinkage coefficient, the variable $x$ in the one-dimension diffusion model can be changed approximately as:

$X \approx(1+\rho(t)) x$

It is assumed that the distribution of incident intensity entered upon the sample is uniform. The contribution of each grating fringe on refractive index modulation can be considered as a total impaction. The total diffracted intensity can be treated as the sum of intensity diffracted by each fringe. The fractional refractive index modulation photoinduced by each grating fringe can be expressed as:

$\Delta n_{i}(X, t) \propto C_{0} \Delta[\text { Polymer }]_{i}(X, t)$

Where, $i$ represents the $i$ th fringe. The total modulation can be described approximately by average of the sum of each refractive index modulation, i.e., average value of modulated polymer's concentration.

$\Delta n(X, t) \propto C_{0} \frac{\sum_{i=1}^{N} \Delta[\text { Polymer }]_{i}(X, t)}{N}$

Where, $N$ is the number of fringe.

\subsection{Simulation}

To illuminate the shrinkage dynamics of grating formation, we solve the equations by finite-difference time-domain method. The dimensionless space domain $\Delta x_{\mathrm{D}}=1-2 \pi / N$, where $N$ is number of sampling points, and the dimensionless time domain $\Delta t_{\mathrm{D}}=0.4\left(\Delta x_{\mathrm{D}}^{2} / R_{D}\right)$ is used ${ }^{19}$. The space of grating is $\Lambda_{0}=0.5 \mu \mathrm{m}$, and the title angle of grating is 6 degree. The dimensionless nonlocal response length $\sigma_{D}=1.0$ is used $^{15}$. The molar-absorption coefficient $\varepsilon=2.1 \times 10^{4}$ $\mathrm{cm}^{2} / \mathrm{mol}$ is used for the simulation ${ }^{18}$. For simplifying, the fractional grating fringes at the center and the edge of grating are respectively described only. Figure 3 illuminated the spatial-temporal dynamic of grating fringes at $\rho_{0}=1 \%$. It is found that the shift of grating fringes at edge of grating is obvious in comparison with the fringes at center of grating, which is ascribed to the cumulating shift of fringes.
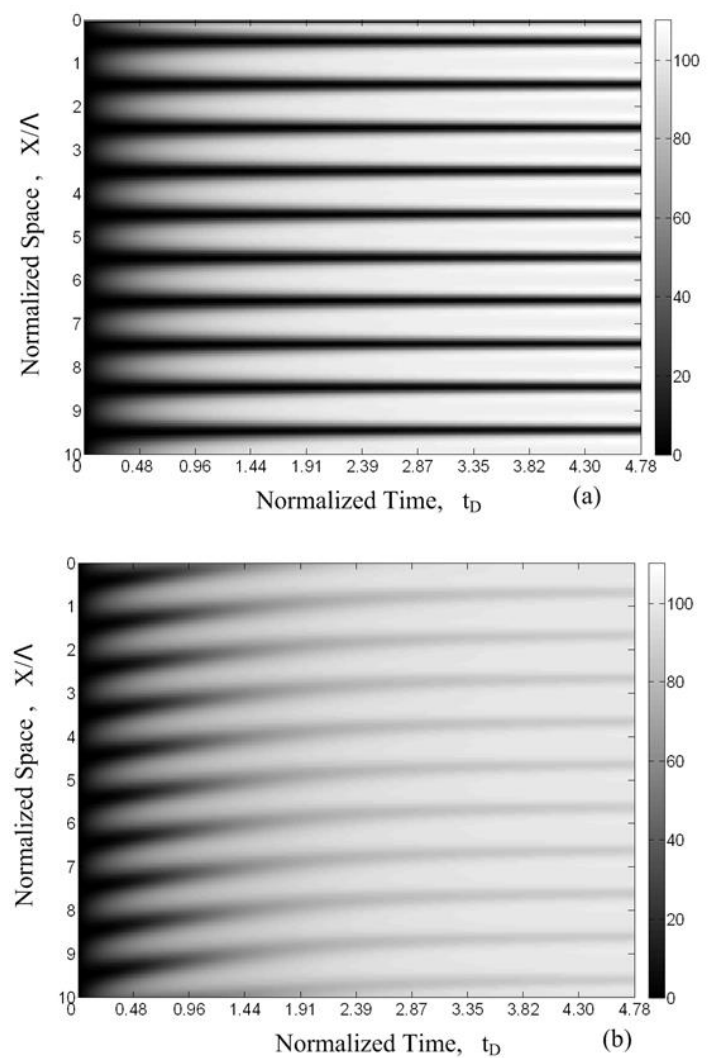

Figure 3. Spatial and temporal dynamics of grating fringes. (a) The fringes at center of grating. (b) The fringes at edge of grating. 


\section{MATEC Web of Conferences}

Figure 4 shows the temporal evolution of refractive index modulation with various shrinkage coefficients. With the increase of shrinkage coefficient, the modulation at steady state and its maximum decrease obviously. It is implied that the high shrinkage leaded to obvious reduction of modulation. The ratio of diffusion rate to polymerization rate $\mathrm{R}=D K^{2} / \kappa I_{a}^{\gamma}$ is a significant parameter for describing the dynamics of grating formation in the photopolymers. According to the experiments of kinetic parameters, the ratio $\mathrm{R} \approx 1$ is obtained in this photopolymer, i.e., the diffusion rate is closed to the polymerization rate. Based on the NPDD model $^{14}$ at $\mathrm{R}=1$, the diffraction efficiency monotonically increase toward a steady saturation and its reduction after reaching saturation is neglectable. Nevertheless, the low diffusion coefficient which leads to $\mathrm{R}<1$ is used to describe the grating formation in the literature ${ }^{15}$, and the final steady value of diffraction efficiency is lower than the peak value, which is ascribed to the formation of polymer gratings which is much faster than the diffusion of monomer molecules. The diffusion coefficient in our material is higher than $10^{-1}-10^{-20} \mathrm{~m}^{2} / \mathrm{s}^{15,20}$, which is ascribed to the difference between the bulk thermal polymerization and the drying polymer-dye mixture in preparation of materials. In this paper, the bulk thermal polymerization results in broad molar mass distribution and high diffusion rate.

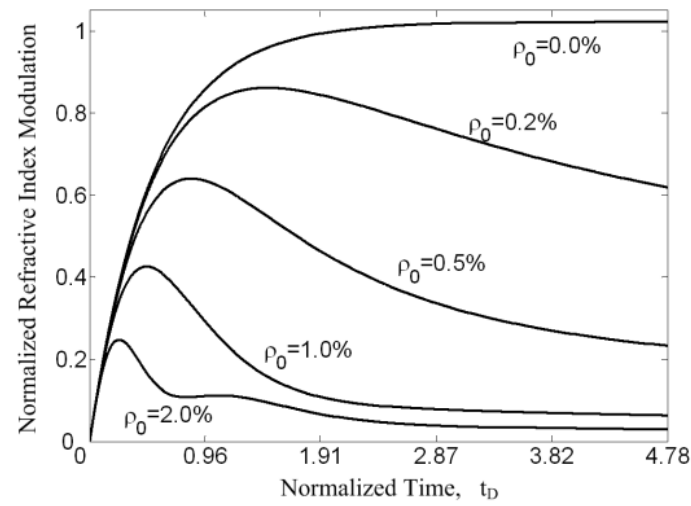

Figure 4. Temporal evolution of refractive index modulation with various shrinkages.

\section{COMPARISON WITH EXPERIMENTS}

The spatial distribution of diffracted intensity as a function of time is schematically shown in Figure 5 The fractional grating fringes instead of the total grating fringes were used to describe the grating formation. At the beginning of grating formation, the distribution of diffracted intensity is closed to uniform. As the increasing shrinkage, the diffracted intensity reduced remarkably at edge of grating. Consequently, the shrinkage process leads to a contrast reduction of holograms and furthermore vanishing edge of holograms. In order to obtain the evident experi- mental results, the hologram is observed by thermal treatment at $40^{\circ} \mathrm{C}$ for enhancing the shrinkage. The inset of Figure 5 showed the evident contrast reduction of hologram. It is demonstrated the validity of theoretical results.

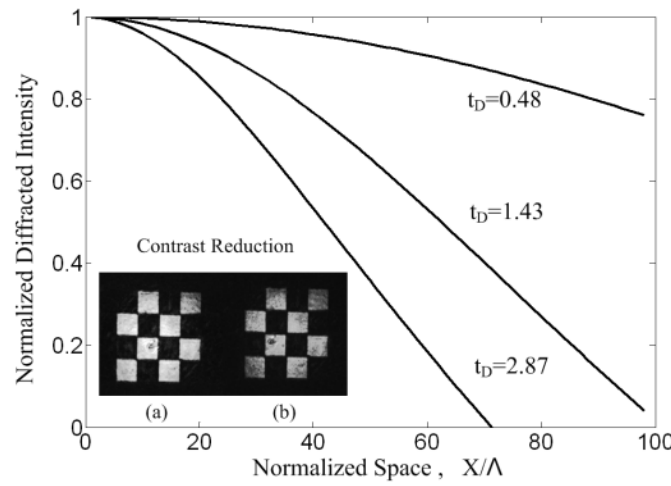

Figure 5. Spatial distribution of diffracted intensity. The inset is experimental results with thermal treatment at $40^{\circ} \mathrm{C}$.
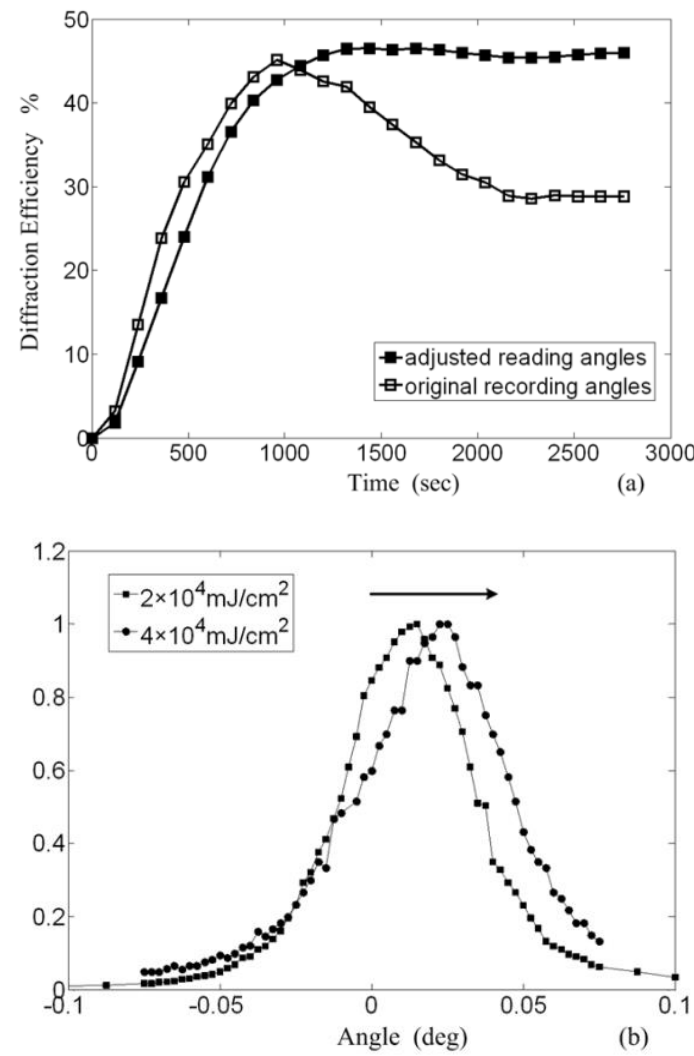

Figure 6. (a) Temporal evolution of diffraction efficiency under consecutive exposure. (b) Angle selectivity curve after various exposures.

The temporal evolution of diffraction efficiency is 
observed under consecutive exposure. The incident intensity is $25.3 \mathrm{mw} / \mathrm{cm}^{2}$. After reaching the maximum of diffraction efficiency, the obvious dropping is observed for reading at original recording angle. However, the diffraction efficiency reaches a steady maximum if the reading angle is adjusted by rotating the sample. The corresponding results are shown in Figure 6(a). Figure 6(b) shows the change of angle selectivity during this process. Consequently, we can conclude that this phenomenon is ascribed to the shrinkage of the materials.

In order to obtain the realistic value of shrinkage coefficient under consecutive exposure, we compared the theoretical results with experiments. An extended model fitted to a growth curve of refractive index modulation is shown in Figure 7. The corresponding shrinkage coefficient $\rho_{0}=0.5 \%$ is extracted. There is low shrinkage coefficient in the PQ-PMMA photopolymer. This result is also consistent with the experimental value in the literature ${ }^{3}$, in which the preparation method of material and bulk thermal polymerization enhances the rigidity of materials. The slight discrepancy between theory and experiment at steady state probably occurs because the residual PQ molecules in and around dark region diffused into bright region and are photoexcited by the subsequent consecutive illumination, which lead to amplification of grating modulation.

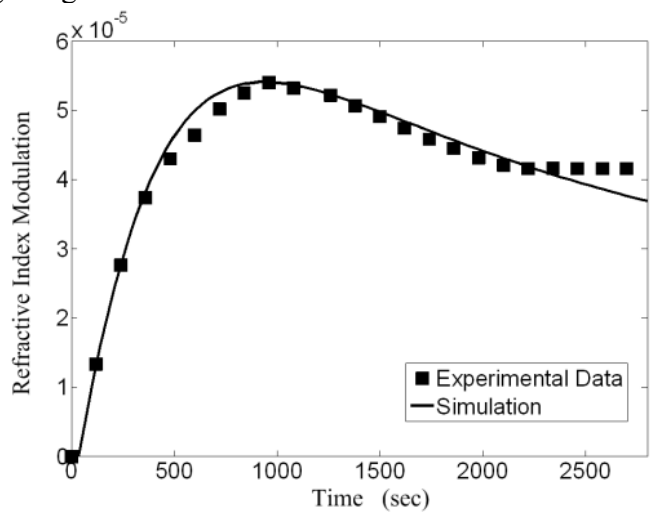

Figure 7. Refractive index modulation as a function of exposure time. The symbols are experimental data and solid line is fitting curve by using extended model and various exposures.

\section{CONCLUSION}

This paper proposed an extended diffusion model by introducing shrinkage coefficient into the model to describe the photopolymerized shrinkage dynamics in PQ-PMMA polymer. To quantitatively simulate, the significant kinetic parameters, polymerization rate and diffusion rate are experimentally determined for the first to our knowledge. For description of shrinkage dynamics, the extended model is then solved numerically by using a finite element technique and the grat- ing evolution is described to take into account the temporal evolution of shrinkage coefficient. The evolution of diffraction efficiency is calculated and the shrinkage coefficient of sample is obtained by comparing the theoretical results with experiments. This is the first paper to simulate the shrinkage dynamics by using diffusion model. It can reflect real situation of grating formation in photopolymers and greatly enhance the applicability of NPDD model.

\section{ACKNOWLEDGEMENT}

This research has been financially supported by National Natural Science Foundation for Young (Grant No. 61307007) and Student's Innovative Training Program (Grant No. 201410060023).

\section{REFERENCES}

[1] Coufal H. et al. 2000. Holographic Data Storage. New York: Springer-Verlag.

[2] Fernández E. et al. 2008. Multiplexed holographic data page storage on a polyvinyl alcohol/acrylamide photopolymer memory. Appl. Opt. 47:4448-4456.

[3] Steckman G. et al. 1998. Characterization of phenanthrenequinone-doped poly(methyl methacrylate) for holographic memory. Opt. Lett. 23:1310-1312.

[4] Lin S. et al. 2000. Phenanthrenequinone-doped poly(methyl methacrylate) photopolymer bulk for volume holographic data storage. Opt. Lett. 25:451-453.

[5] Matusevich V. et al. 2008. Holographic volume absorption grating in glass-like polymer recording material. Opt. Express 16:1552-1558

[6] Luo Y. et al. 2008. Optimization of multiplexed holographic gratings in PQ-PMMA for spectral-spatial imaging filters. Opt. Lett. 33:566-568.

[7] Zhao C. et al. 1997. Shrinkage-corrected volume holograms based on photopolymeric phase media for surface-normal optical interconnects. Appl. Phys. Lett. 71:1464-1466.

[8] Chen J.H. et al. 2002. Shrinkage and refractive-index shift-corrected volume holograms for optical interconnects. Appl. Phys. Lett. 81:1387-1389.

[9] Dhar L. et al. 1998. Temperature-induced changes in photopolymer volume holograms. Appl. Phys. Lett. 73:1337-1339.

[10]Pandey N. et al. 2008. Technique for characterization of dimensional changes in slanted holographic gratings by monitoring the angular selectivity profile. Opt. Lett. 33:1981-1983.

[11] Gallo J \& Verber C. 1994. Model for the effects of material shrinkage on volume holograms. Appl. Opt. 33:6797-6800.

[12]Hsieh M \& Hsu K. 2001. Grating detuning effect on holographic memory in photopolymers. Opt. Eng. 40:2125-2133.

[13]Wu S \& Glytsis E. 2004. Characteristics of DuPont photopolymers for slanted holographic grating formations. J. Opt. Soc. Am. B 21:1722-1731. 


\section{MATEC Web of Conferences}

[14] Sheridan J \& Lawrence J. 2000. Non-local response diffusion model of holographic recording in photopolymer. J. Opt. Soc. Am. A 17:1108-1114.

[15]Tolstik E. et al. 2008. Non-local response in glass-like polymer storage materials based on poly (methylmethacrylate) with distributed phenanthrenequinone. Opt. Express 16:11253-11258.

[16] Moreau V. et al. 2002. Characteristics of DuPont photopolymer: determination of kinetic parameters in a diffusion model. Appl. Opt. 41:3427-3435.

[17]Fernandez-Pousa C. et al. 2000. Holographic determination of the irradiance dependence of linear-chain polymerization rates in photopolymer dry films. Appl. Phys. B 70:537-542.
[18]Liu H. et al. 2009. Characteristics of holographic scat tering and its application in determining kinetic parameters in PQ-PMMA photopolymer. Appl. Phys. B 95:513-518.

[19]Wu S \& Glytsis E. 2003. Holographic grating formation in photopolymers: analysis and experimental results based on a nonlocal diffusion model and rigorous coupled-wave analysis. J. Opt. Soc. Am. B 20:1177-1188.

[20]Popov A. et al. 2000. Spectrally selective holographic optical elements based on a thick polymer medium with diffusional amplification. J. Opt. A: Pure Appl. Opt. 2:494-499. 